\title{
Reviews and Sensitization About Cancer in Algeria
}

\author{
Type of article: conference Abstract
}

\author{
Kaouel Meguenni \\ University of Tlemcen \\ k.meguenni@gmail.com
}

\begin{abstract}
Having cancer data is a necessity for several reasons. Researchers need accurate data and updates on cancer for the study of possible causes, managers for decisions concerning the acquisition of equipment and/or development activities or programs for treatment, screening and prevention, and the health authorities to investigate cancer groups, their trends and their causes. Where does the data come, and how is it collected? In fact, all these cancer statistics are collected and provided by the Cancer Registry, from clinical records, and from biological and care institutions. By definition, a cancer registry is an information system and an epidemiological surveillance tool for the collection, storage, management, data analysis, and dissemination on incident cases of cancer, covering a geographically defined population. The cancer registry is an essential tool of any program against cancer. Its primary objective is, indeed, the description of the epidemiological situation, the study of trends and geographic distribution of cases, but it is essential to other areas in particular:

- Contribution to the study of the natural history of the disease

- Contribution to the etiological and risk factors

- $\quad$ Clinical research on treatment plans and prognosis

- Identification of needs in health planning and development of prevention strategies

Cancer registries are a primary source for cancer control strategies; they can be a source of cohort studies and clinical trials, and perhaps, most importantly, the starting point for the analysis of survival, if death registration in the civil registry office followed a comprehensive approach, standardized and reliable through the establishment of the mandatory death certificate as recommended by WHO, and provided for the causes of a death surveillance program. The registration cancer process of continuous and systematic collection of data on the incidence of cancer is the result of sustained and continuous effort of a team but also the institution's commitment.
\end{abstract}

Keywords: Cancer Registry, Data analyzes, Statistics, Epidemiology, Algeria.

\section{Declaration of conflicts}

This article was presented at ICHSMT'16 as a key notes.

\section{Authors' biography}

Professor Dr. Kaouel Meguenni is cancer lab director, professor of epidemiology at University of Tlemcen, head of the Epidemiology Department at Universitary Hospital of Tlemcen

\section{References}

\section{No reference}

